\title{
Tumor Budding and Poorly Differentiated Clusters in Small Intestinal Adenocarcinoma
}

\author{
Sun-Young Jun ${ }^{1, *(\mathbb{D}}$, Joon-Yong Chung ${ }^{2}{ }^{\mathbb{D}}$, Nara Yoon ${ }^{1}$, Eun Sun Jung ${ }^{3}$, Young-Ha Oh ${ }^{4} \mathbb{C}$ \\ and Seung-Mo Hong ${ }^{5, *}$ \\ 1 Department of Pathology, Incheon St. Mary's Hospital, College of Medicine, \\ The Catholic University of Korea, Seoul 21431, Korea; waxggul@gmail.com \\ 2 Laboratory of Pathology, Center for Cancer Research, National Cancer Institute, \\ National Institutes of Health, Bethesda, MD 20892, USA; chungjo@mail.nih.gov \\ 3 Department of Pathology, Eunpyeong St. Mary's Hospital, College of Medicine, \\ The Catholic University of Korea, Seoul 03312, Korea; esjung@catholic.ac.kr \\ 4 Department of Pathology, Hanyang University College of Medicine, Guri 11923, Korea; yhoh@hanyang.ac.kr \\ 5 Department of Pathology, Asan Medical Center, University of Ulsan College of Medicine, Seoul 05505, Korea \\ * Correspondence: drssun@empal.com (S.-Y.J.); smhong28@gmail.com (S.-M.H.); Tel.: +82-32-280-5518 (S.-Y.J.); \\ $+82-2-3010-4558$ (S.-M.H.)
}

Received: 8 July 2020; Accepted: 4 August 2020; Published: 6 August 2020

check for updates

\begin{abstract}
The clinicopathologic and prognostic significances of tumor budding (TB) and poorly-differentiated clusters (PDC) have not been investigated in small intestinal adenocarcinomas (SIACs). In 236 surgically-resected SIACs, we counted TB (single cells or clusters $\leq 4$ tumor cells) and PDC (clusters $\geq 5$ tumor cells) at the peritumoral-invasive front ( $p$ ) and in the intratumoral area (i) independently to classify as grade-1 $(\leq 4)$, grade-2 (5-9), or grade-3 $(\geq 10)$. Consequently, grades-2 and -3 were considered high-grade. High-pTB, -iTB, -pPDC, and -iPDC were observed in 174 (73.7\%), 129 (54.7\%), 118 (50.0\%), and 85 (36.0\%) cases, respectively. High-TB/PDCs were more frequently observed in tumors with high-grade, higher T- and N-categories and stage grouping, and perineural or lymphovascular invasion. Patients with high-TB/PDC had a shorter survival than those with low-TB/PDC. In a multivariate analysis, high-pTB, nonintestinal type, high N-category, retroperitoneal seeding, and microsatellite-stable were worse independent-prognostic predictors. Subgroup analysis demonstrated that patients with high-pTB showed worse survival (median: 42.5 months) than those with low-pTB (133.7 months; $p=0.007$ ) in the lower stage (stages I-II) group. High-TB/PDC, both in peritumoral and intratumoral localizations, were associated with aggressive behaviors in SIACs. High-pTB can be used as an adverse prognostic indicator in SIAC patients, especially when patients are in early disease stages.
\end{abstract}

Keywords: small intestine; adenocarcinoma; tumor budding; poorly differentiated clusters; prognosis

\section{Introduction}

Small intestinal adenocarcinoma (SIAC) is very rare, compromising less than $3 \%$ of gastrointestinal (GI) malignancies [1]. In the United States, the overall incidence of SIAC has been increasing slowly since the early 1990s [2], and an estimated 11,110 new cases are expected to be diagnosed in 2020 [3]. In Korea, 894 new cases were diagnosed with SIAC in 2016 [4].

SIAC tends to be found at an advanced stage due to nonspecific symptoms and difficulty detecting the tumors [5]. Moreover, there are no appropriate screening programs, even for potentially high-risk individuals [6]. To make matters worse, few studies have been published to help guide the management of SIAC owing to the rarity of the tumors [6]. Although the National Comprehensive Cancer Network (NCCN) has recently published guidelines for the treatment of SIAC [7], management of SIAC has 
been historically extrapolated from colorectal cancer (CRC). Patient outcomes for SIAC are inferior to those for CRC at all stages of diagnosis [1,2].

Previous studies on SIACs have concentrated on comparisons with CRCs because of the anatomical proximity and histopathological similarities [5,8-11]. As a result, few prognostic factors have been identified in SIACs such as tumor location (duodenum vs. jejunum and ileum), microsatellite instability (MSI), tumor-infiltrating lymphocytes (TILs), and KRAS or BRAF mutations [9-11]. Kim et al. demonstrated that the complete epithelial-mesenchymal transition (EMT) phenotype, exhibiting an absence of E-cadherin expression and presence of vimentin and/or fibronectin expression, represented a poor clinical outcome in patients with SIAC [12].

Tumor budding (TB), both intratumoral (buds in the tumor center, iTB) and peritumoral (buds at the invasive front, pTB), has been well known to be a morphologic manifestation of EMT $[13,14]$. TB is defined as a single tumor cell or a small tumor cluster consisting of as many as four tumor cells [15]. Recently, the International Consensus Conference on Tumor Budding (ITBCC) recommended an evidence-based, standardized scoring system for TB to be used in CRC, which can be categorized into low ( $0-4$ buds), intermediate (5-9 buds), and high (10 or more buds) levels based on counting buds on hematoxylin and eosin ( $\mathrm{H}$ and $\mathrm{E}$ ) slides in one hotspot of 20× objective lens [13]. Over the past decade, there was mounting evidence of the adverse effect of TB in CRCs and other GI malignancies, most of which revealed PTB as a strong predictor of lymphovascular invasion, tumor recurrence, nodal and distant metastases, and poor patients' survival [13,16-24]. On the other hand, Lugli et al. found a strong relationship between $\mathrm{PTB}$ and iTB and emphasized their similar prognostic significance in CRCs [23].

Poorly-differentiated clusters (PDC) were first described in 2008 and similar to TB, they were a highly reproducible and relevant factor for predicting the prognosis and metastatic risk of patients with CRCs [25-27]. PDC, defined as solid cancer nests comprising five or more cancer cells and lacking glandular formation, have shared an identical grading system with TB since $2012[15,28]$. Ueno et al. proposed that PDC can predict prognostic outcome in CRC patients more accurately than TB [28]. Tumor grading via PDC quantification was shown to be reliable and more informative than tumor-node-metastasis (TNM) staging for predicting prognosis in CRC [29]. Bertoni et al. suggested that PPDC and iPDC are biologically different based on their discordant pattern of $\beta$-catenin and E-cadherin expression [30]. Meanwhile, high levels of PDC in tumors may be linked to RAS oncogenic mutations inducing dedifferentiation of the EMT process [31]. However, the clinicopathologic and prognostic significances of TB and PDC have not been investigated in SIACs.

In this study, we analyzed TB and PDC in both peritumoral and intratumoral regions in detail and verified their associations with clinicopathologic variables and prognostic values in SIAC.

\section{Materials and Methods}

\subsection{Tissue Samples}

A total of 236 surgically resected primary SIAC cases were enrolled, including 231 previously reported cases [9] and 4 additional cases. Primary duodenal, jejunal, and ileal carcinomas were included in this study. On the contrary, we excluded carcinomas extending from the ampulla of Vater into the small intestines. This study was approved by the Institutional Review Board of Incheon St. Mary's Hospital (OC14OIMI0133), and patient consent was waived due to the retrospectively obtained and anonymized data of this study.

Clinicopathologic information collected as part of a previous study was updated [9]. Histologic subtypes were classified by the 5th edition of the World Health Organization (WHO) classification [1]. A tumor containing extracellular mucin greater than $50 \%$ of the tumor volume was designated as mucinous carcinoma [1]. A signet ring cell carcinoma was defined when more than $50 \%$ of the tumor cells had prominent intracytoplasmic mucin, typically with the displacement of the nuclei [15]. T- and $\mathrm{N}$-categories and stage grouping were evaluated according to the 8th American Joint Committee on 
Cancer (AJCC) staging system [32]. In addition, we classified SIACs into intestinal and nonintestinal immune-phenotypes based on the combined CDX2 and MUC1 expression patterns, as previously reported [33]. SIAC cases expressing CDX2+/MUC1- were considered intestinal immune-phenotypes, while the other SIACs with CDX2-/MUC1+, CDX2+/MUC1+, and CDX2-/MUC1-immunoreactivities were categorized as nonintestinal [33]. KRAS and BRAF genotyping results and microsatellite instability (MSI) status obtained from the previous studies were also included [9,11]. Briefly, mutations in codons 12 and 13 of KRAS and codon 600 of BRAF exon 15 were analyzed by Sanger sequencing using formalin-fixed paraffin-embedded tissue blocks [11]. For MSI status assessment, five quasi-monomorphic mononucleotide repeats, including BAT25, BAT26, NR21, NR24, and NR27, were analyzed using a single multiplex polymerase chain reaction [9]. According to the National Cancer Institute (NCI) guidelines, tumors were divided into MSI-high (instability at $\geq 2$ mononucleotide loci), MSI-low (instability at a single mononucleotide locus), and microsatellite stable (MSS; no instability at any of the loci tested) [9].

\section{2. $T B$ and $P D C$}

Representative $\mathrm{H}$ and E-stained, full-faced sections containing the deepest part of the invasive front of the tumor were selected. TB was defined as a single cell or clusters of as many as 4 tumor cells, whereas PDC was defined as clusters of 5 or more tumor cells without glandular formation (Figure 1A-D) [15]. TB and PDC were independently counted at the peritumoral invasive front (p) and in the intratumoral area (i) by two experienced GI pathologists (S.-Y.J. and N.Y.) blinded to clinicopathologic information using an Olympus BX-53 microscope (Olympus, Tokyo, Japan). Discrepancies were resolved by simultaneous reevaluation and discussion. At least 10 individual fields at medium power (10× objective) were scanned to identify the "hotspot" of TB and PDC, and TB and PDC were counted in one hotspot of $20 \times$ objective (area, $0.785 \mathrm{~mm}^{2}$ ) to be categorized into grade- 1 (0 to 4 ), grade-2 (5 to 9), or grade-3 ( $\geq 10)$, according to the ITBCC criteria [13]. Consequently, grade- 2 or -3 were considered high levels of TB and PDC, independently (Figure 1).

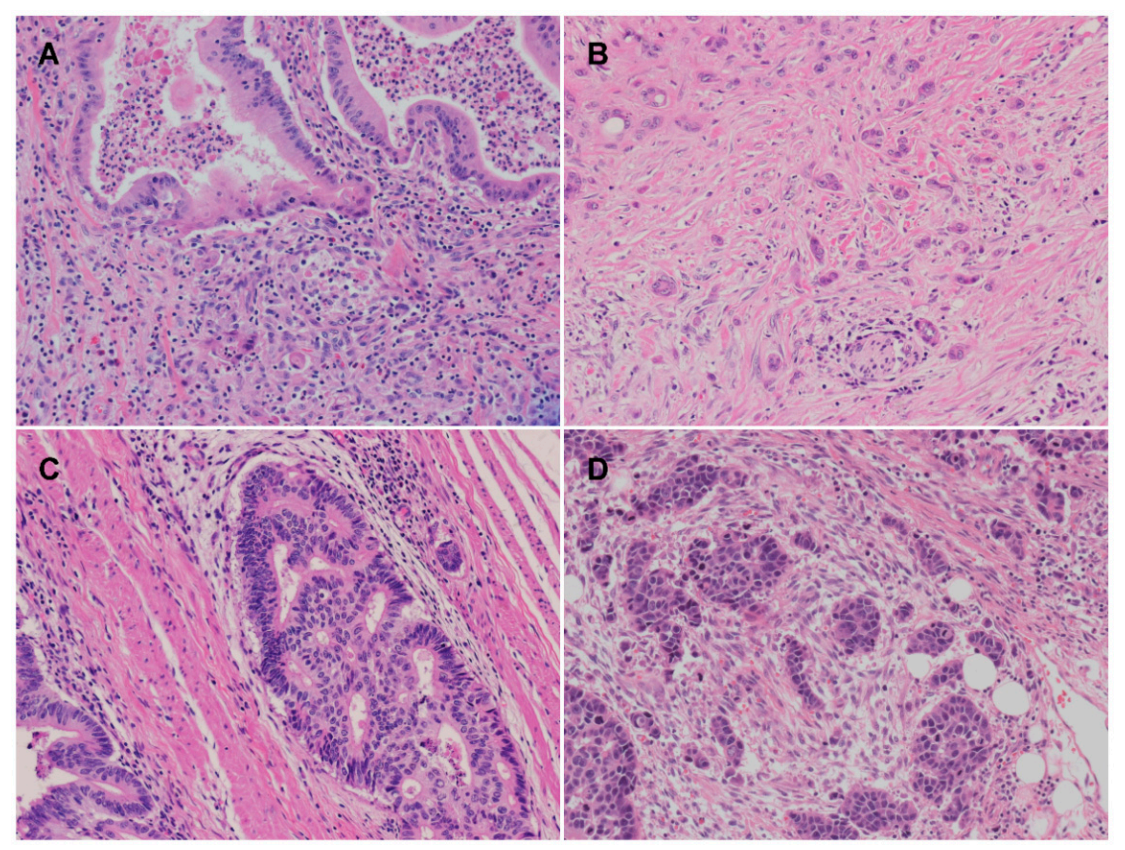

Figure 1. Representative images of tumor budding (TB) and poorly-differentiated clusters (PDC). $(\mathbf{A}, \mathbf{B})$ TB is defined as a single tumor cell or as a tumor cell cluster of as many as 4 cancer cells. In contrast, (C,D) a tumor cell cluster containing 5 or more cancer cells and lacking glandular formation is categorized as PDC. Based on the counting levels, they are independently categorized as (A) low TB, (B) high TB, (C) low PDC, and (D) high PDC (all, H and E, 200× magnification). 


\subsection{Statistical Analysis}

All statistical analyses were performed using SPSS software (version 17.0; SPSS Inc., Chicago, IL, USA). Mean values were compared by the Student's $t$ test or simple analysis of variance. Associations between clinicopathologic factors were assessed using $\chi^{2}$ and Fisher's exact test. Logistic regression models were used to evaluate multivariate associations. Overall survival probabilities were plotted with the Kaplan-Meier method, and the significance of differences in survival probabilities were probed using a log-rank test. Univariate and multivariate survival analyses were performed using the Cox proportional hazards modeling. A $p$ value of $<0.05$ was considered statistically significant.

\section{Results}

\subsection{Clinicopathologic Features}

The mean age of the SIAC patients was $59.6 \pm 12.7$ years, and the male to female ratio was 1.8. Tumor size was evaluated in 234 cases, and the mean tumor size was $4.3 \pm 0.2 \mathrm{~cm}$. The median follow-up period after surgical resection was 25.3 months (range, 0.3-168.4 months). SIACs were located in the duodenum in 142 cases $(60.2 \%)$, the jejunum in $61(25.8 \%)$, and the ileum in $33(14.0 \%)$. According to the AJCC staging scheme, 5 tumors were categorized as Tis $(2.1 \%), 14$ as T1 $(5.9 \%), 14$ as T2 $(5.9 \%), 70$ as T3 $(29.7 \%)$, and 133 as T4 (56.4\%). Lymph nodes were examined in 219 cases, and half of them were N0 (111 cases, 50.7\%). Nodal metastases were seen in 108 cases, including $55 \mathrm{~N} 1(25.1 \%)$ and $53 \mathrm{~N} 2(24.2 \%)$. Consequently, the tumors were classified into stages 0 ( 5 cases, $2.3 \%)$, I (23, 10.5\%), IIA $(33,15.1 \%)$, IIB $(50,22.8 \%)$, IIIA (55, 25.1\%), and IIIB (53, 24.2\%). The combined expression patterns of CDX2 and MUC1 were assessed in 230 interpretable tumors [33]. Among 230 SIACs, 153 (66.5\%) cases were classified as nonintestinal and $77(33.5 \%)$ were intestinal. The mutation status of KRAS and $B R A F$ was evaluated in 190 and 178 patients, respectively, as previously described [9]. KRAS and BRAF mutations were identified in $32.1 \%(61 / 190)$ and $1.1 \%$ (2/178) of cases, respectively [11]. MSI analysis was available in 230 SIAC cases. MSI was found in 50 cases (21.7\%), all of which were MSI-high [9].

pTB was variably observed in $88.1 \%$ (208/236), of which levels were up to 60 (mean, $11.4 \pm 11.0$ ). iTB was identified in 78.0\% (184/236), ranging up to 600 (mean, $12.9 \pm 6.0$ ). pPDC and iPDC were seen in 86.4\% (204/236; maximum, 36; mean, $5.9 \pm 5.3)$ and 77.1\% (182/236; maximum, 30; mean, $4.6 \pm 5.1$ ), respectively. Based on the counting level, two thirds of the tumors $(155 / 236,65.7 \%)$ were categorized as grade- 3 of pTB. Meanwhile, 62 cases were grade- $1(26.3 \%)$ and 19 were as grade- $2(8.0 \%)$ of pTB. In iTB, half of the tumors were grade- 1 (107 cases, $45.3 \%)$, and grades- 2 and -3 were seen in $32(13.6 \%)$ and 97 $(41.1 \%)$ cases, respectively. In pPDC, $118(50.0 \%)$ cases of the tumors were grade- $1,45(19.1 \%)$ were grade-2, and 73 (30.9\%) were grade-3. iPDC was classified into grade- 1 in $151(64.0 \%)$ cases, grade- 2 in $34(14.4 \%)$, and grade- 3 in $51(21.6 \%)$. After merging cases with grades- 2 and -3 and categorizing them as high level, high pTB, iTB, pPDC, and iPDC were observed in 174 (73.7\%), $129(54.7 \%), 118(50.0 \%)$, and $85(36.0 \%)$ cases, respectively.

\subsection{Associations between Clinicopathologic Factors and TB and PDC}

The associations between clinicopathologic factors and TB and PDC are summarized in Tables 1 and 2, respectively. In TB, high levels of both $\mathrm{PTB}$ and iTB were associated with aggressive behaviors of SIACs, including nodular or infiltrative growth pattern, high tumor grade, and perineural or lymphovascular invasion ( $p<0.001$, all), pancreatic invasion $(p=0.007$ and $p=0.022$, respectively), nodal metastasis ( $p<0.001$ and $p=0.002$, respectively), and higher T- ( $p<0.001$, both) and N-categories $(p<0.001$ and $p=0.006$, respectively) and stage grouping ( $p<0.001$ and $p=0.006$, respectively). Nonintestinal types of SIACs more frequently exhibited high levels of pTB and iTB $(p<0.001$, both). On the other hand, high PTB was more commonly observed in patients with radiotherapy $(p=0.015)$, whereas high iTB was in signet ring cell carcinoma and undifferentiated carcinoma $(p=0.020)$ and in tumors with retroperitoneal seeding $(p=0.042)$. 
Table 1. Association between Clinicopathologic Factors and TB Status in SIAC.

\begin{tabular}{|c|c|c|c|c|c|c|c|}
\hline \multirow{2}{*}{\multicolumn{2}{|c|}{ Variable }} & \multicolumn{3}{|c|}{ pТВ, $N(\%)$} & \multicolumn{3}{|c|}{ iTB, $N(\%)$} \\
\hline & & Low & High & $p$ & Low & High & $p$ \\
\hline \multicolumn{2}{|c|}{ No. of patients } & $62(26)$ & $174(74)$ & & $107(45)$ & $129(55)$ & \\
\hline \multicolumn{2}{|c|}{ Age (mean $\pm S D$, years) } & $57.7 \pm 14.2$ & $60.3 \pm 12.3$ & 0.156 & $58.6 \pm 13.0$ & $60.5 \pm 12.7$ & 0.247 \\
\hline \multicolumn{2}{|c|}{ Size $(\text { mean } \pm S D, c m)^{a}$} & $4.5 \pm 2.5$ & $4.3 \pm 2.5$ & 0.616 & $4.5 \pm 2.4$ & $4.2 \pm 2.6$ & 0.469 \\
\hline \multirow{2}{*}{ Age (years) } & $\leq 50$ & $19(34)$ & $36(66)$ & 0.111 & $26(47)$ & $29(53)$ & 0.742 \\
\hline & $>50$ & $43(24)$ & $138(76)$ & & $81(45)$ & $100(55)$ & \\
\hline \multirow[t]{2}{*}{ Sex } & Male & $40(26)$ & $112(74)$ & 0.983 & $63(41)$ & $89(59)$ & 0.106 \\
\hline & Female & $22(26)$ & $62(74)$ & & $44(52)$ & $40(48)$ & \\
\hline \multirow[t]{3}{*}{ Growth pattern ${ }^{a}$} & Polypoid & $27(61)$ & $17(39)$ & $<0.001$ & $35(80)$ & $9(20)$ & $<0.001$ \\
\hline & Nodular & $4(23)$ & $13(77)$ & & $7(41)$ & $10(59)$ & \\
\hline & Infiltrative & $28(17)$ & $139(83)$ & & $59(35)$ & $108(65)$ & \\
\hline \multirow[t]{2}{*}{ Location } & Proximal (duodenum) & $43(30)$ & $99(70)$ & 0.085 & $69(49)$ & $73(51)$ & 0.217 \\
\hline & Distal (jejunum, ileum) & $19(20)$ & $75(80)$ & & $38(40)$ & $56(60)$ & \\
\hline \multirow[t]{4}{*}{ Histologic subtype } & Tubular & $58(28)$ & $152(72)$ & 0.256 & $96(46)$ & $114(54)$ & 0.020 \\
\hline & Mucinous & $4(36)$ & $7(64)$ & & $8(73)$ & $3(27)$ & \\
\hline & Signet ring cell & 0 & $4(100)$ & & 0 & $4(100)$ & \\
\hline & Undifferentiated & 0 & $5(100)$ & & 0 & $5(100)$ & \\
\hline Immunophenotype ${ }^{a}$ & Nonintestinal & $26(17)$ & $127(83)$ & & $54(35)$ & $99(65)$ & \\
\hline \multirow[t]{2}{*}{ Differentiation } & Low (Well to moderately) & $59(32)$ & $128(68)$ & $<0.001$ & $100(54)$ & $87(46)$ & $<0.001$ \\
\hline & High (Poorly or undifferentiated) & $3(6)$ & $46(94)$ & & $7(14)$ & $42(86)$ & \\
\hline \multirow[t]{2}{*}{ Pancreas invasion } & Absent & $48(32)$ & $101(68)$ & 0.007 & $76(51)$ & $73(49)$ & 0.022 \\
\hline & Present & $14(16)$ & $73(84)$ & & $31(36)$ & $56(64)$ & \\
\hline \multirow[t]{2}{*}{ Other loop invasion } & Absent & $62(27)$ & $168(73)$ & 0.345 & $106(46)$ & $124(54)$ & 0.225 \\
\hline & Present & 0 & $6(100)$ & & $1(17)$ & $5(83)$ & \\
\hline \multirow[t]{2}{*}{ Retroperitoneal seeding } & Absent & $61(28)$ & $160(72)$ & 0.125 & $104(47)$ & $117(53)$ & 0.042 \\
\hline & Present & $1(7)$ & $14(93)$ & & $3(20)$ & $12(80)$ & \\
\hline \multirow[t]{2}{*}{ Lymphovascular invasion } & Absent & $52(43)$ & $68(57)$ & $<0.001$ & $72(60)$ & $48(40)$ & $<0.001$ \\
\hline & Present & $10(9)$ & $106(91)$ & & $35(30)$ & $81(70)$ & \\
\hline \multirow[t]{2}{*}{ Perineural invasion } & Absent & $55(35)$ & $104(65)$ & $<0.001$ & $87(55)$ & $72(45)$ & $<0.001$ \\
\hline & Present & $7(9)$ & $70(91)$ & & $20(26)$ & $57(74)$ & \\
\hline Resection margin status ${ }^{a}$ & No involvement & $54(25)$ & $159(75)$ & 0.058 & $98(46)$ & $115(54)$ & 1.000 \\
\hline
\end{tabular}


Table 1. Cont.

\begin{tabular}{|c|c|c|c|c|c|c|c|}
\hline \multirow{2}{*}{\multicolumn{2}{|c|}{ Variable }} & \multicolumn{3}{|c|}{ pTB, $N(\%)$} & \multicolumn{3}{|c|}{ iTB, $N(\%)$} \\
\hline & & Low & High & $p$ & Low & High & $p$ \\
\hline \multirow{3}{*}{ Nodal metastasis ${ }^{\text {a }}$} & Cancer involvement & $5(56)$ & $4(44)$ & & $4(44)$ & $5(56)$ & \\
\hline & No & $48(43)$ & $63(57)$ & $<0.001$ & $62(56)$ & $49(44)$ & 0.002 \\
\hline & Yes & $11(10)$ & $97(90)$ & & $38(35)$ & $70(65)$ & \\
\hline \multirow[t]{2}{*}{$\mathrm{T}_{\text {category }} \mathrm{b}$} & Low (T1-T2) & $21(75)$ & $7(25)$ & $<0.001$ & $25(89)$ & $3(11)$ & $<0.001$ \\
\hline & High (T3-T4) & $36(18)$ & $167(82)$ & & $77(38)$ & $126(62)$ & \\
\hline \multirow[t]{3}{*}{$\mathrm{N}$ category ${ }^{\mathrm{a}}$} & N0 & $48(43)$ & $63(57)$ & $<0.001$ & $62(56)$ & $49(44)$ & 0.006 \\
\hline & N1 & $4(7)$ & $51(93)$ & & $17(31)$ & $38(69)$ & \\
\hline & $\mathrm{N} 2$ & $7(13)$ & $46(87)$ & & $21(40)$ & $32(60)$ & \\
\hline \multirow[t]{2}{*}{ Stage grouping a,b } & Low (Stages I-II) & $43(41)$ & $63(59)$ & $<0.001$ & $57(54)$ & $49(46)$ & 0.006 \\
\hline & High (Stage III) & $11(10)$ & $97(90)$ & & $38(35)$ & $70(65)$ & \\
\hline \multirow[t]{2}{*}{ Chemotherapy ${ }^{a}$} & No & $46(31)$ & $103(69)$ & 0.062 & $70(47)$ & $79(53)$ & 0.530 \\
\hline & Yes & $16(19)$ & $66(81)$ & & $35(43)$ & $47(57)$ & \\
\hline \multirow[t]{2}{*}{ Radiation therapy $^{\text {a }}$} & No & $60(30)$ & $143(70)$ & 0.015 & $95(47)$ & $108(53)$ & 0.339 \\
\hline & Yes & $2(7)$ & $25(93)$ & & $10(37)$ & $17(63)$ & \\
\hline \multirow[t]{2}{*}{ KRAS genotype ${ }^{\mathrm{a}}$} & Wild type & $35(27)$ & $94(73)$ & 0.172 & $57(44)$ & $72(56)$ & 0.839 \\
\hline & Mutated & $11(18)$ & $50(82)$ & & $26(43)$ & $35(57)$ & \\
\hline \multirow[t]{2}{*}{$B R A F$ genotype $^{\mathrm{a}}$} & Wild type & $44(25)$ & $132(75)$ & 1.000 & $80(45)$ & $96(55)$ & 0.502 \\
\hline & Mutated & 0 & $2(100)$ & & 0 & $2(100)$ & \\
\hline \multirow[t]{2}{*}{ MSI status ${ }^{a}$} & MSS & $46(25)$ & $139(75)$ & 0.462 & $81(44)$ & $104(56)$ & 0.433 \\
\hline & MSI-high & $15(30)$ & $35(70)$ & & $25(50)$ & $25(50)$ & \\
\hline
\end{tabular}

Small intestinal adenocarcinoma (SIAC); standard deviation (SD); microsatellite stable (MSS); microsatellite instability (MSI); significant $p$-values in bold; ${ }^{2}$ calculated using only patients with adequate data; ${ }^{b}$ excluded patients with Tis and stage 0. 
Table 2. Association between Clinicopathologic Factors and PDC Status in SIAC.

\begin{tabular}{|c|c|c|c|c|c|c|c|}
\hline \multirow{2}{*}{\multicolumn{2}{|c|}{ Variable }} & \multicolumn{3}{|c|}{$\mathrm{pPDC}, N(\%)$} & \multicolumn{3}{|c|}{ iPDC, $N(\%)$} \\
\hline & & Low & High & $p$ & Low & High & $p$ \\
\hline \multicolumn{2}{|c|}{ No. of patients } & $118(50)$ & $118(50)$ & & $151(64)$ & $85(36)$ & \\
\hline \multicolumn{2}{|c|}{ Age (mean \pm SD, years) } & $58.2 \pm 14.1$ & $61.1 \pm 11.4$ & 0.082 & $59.3 \pm 13.3$ & $60.2 \pm 12.0$ & 0.599 \\
\hline \multicolumn{2}{|c|}{ Size $(\text { mean } \pm S D, c m)^{a}$} & $4.4 \pm 2.5$ & $4.3 \pm 2.5$ & 0.712 & $4.2 \pm 2.3$ & $4.5 \pm 2.9$ & 0.492 \\
\hline \multirow[t]{2}{*}{ Age (years) } & $\leq 50$ & $36(66)$ & $19(34)$ & 0.009 & $37(67)$ & $18(33)$ & 0.562 \\
\hline & $>50$ & $82(45)$ & 99 (55) & & $114(63)$ & $67(37)$ & \\
\hline \multirow[t]{2}{*}{ Sex } & Male & $79(52)$ & $73(48)$ & 0.415 & $97(64)$ & $55(36)$ & 0.943 \\
\hline & Female & $39(46)$ & $45(54)$ & & $54(64)$ & $30(36)$ & \\
\hline \multirow[t]{3}{*}{ Growth pattern ${ }^{a}$} & Polypoid & $34(77)$ & $10(23)$ & $<0.001$ & $38(86)$ & $6(14)$ & 0.002 \\
\hline & Nodular & $9(53)$ & $8(47)$ & & $11(65)$ & $6(35)$ & \\
\hline & Infiltrative & $73(44)$ & $94(56)$ & & $96(58)$ & $71(42)$ & \\
\hline \multirow[t]{2}{*}{ Location } & Proximal (duodenum) & $73(51)$ & $69(49)$ & 0.595 & $95(67)$ & $47(33)$ & 0.251 \\
\hline & Distal (jejunum, ileum) & $45(48)$ & $49(52)$ & & $56(60)$ & $38(40)$ & \\
\hline \multirow[t]{5}{*}{ Histologic subtype } & Tubular & $108(51)$ & $102(49)$ & 0.134 & $134(64)$ & $76(36)$ & 0.069 \\
\hline & Mucinous & $5(45)$ & $6(55)$ & & $8(73)$ & $3(27)$ & \\
\hline & Signet ring cell & $2(50)$ & $2(50)$ & & $4(100)$ & 0 & \\
\hline & Undifferentiated & $3(60)$ & $2(40)$ & & $4(80)$ & $1(20)$ & \\
\hline & Medullary & 0 & $6(100)$ & & $1(17)$ & $5(83)$ & \\
\hline \multirow{2}{*}{ Immunophenotype ${ }^{a}$} & Intestinal & $46(60)$ & $31(40)$ & 0.029 & $57(74)$ & $20(26)$ & 0.018 \\
\hline & Nonintestinal & $68(44)$ & $85(56)$ & & $89(58)$ & $64(42)$ & \\
\hline \multirow[t]{2}{*}{ Differentiation } & Low (Well to moderately) & $103(55)$ & $84(45)$ & 0.002 & $132(71)$ & $55(29)$ & $<0.001$ \\
\hline & High (Poorly or undifferentiated) & $15(31)$ & $34(69)$ & & $19(39)$ & $30(61)$ & \\
\hline \multirow[t]{2}{*}{ Pancreas invasion } & Absent & $83(56)$ & $66(44)$ & 0.022 & $101(68)$ & $48(32)$ & 0.111 \\
\hline & Present & $35(40)$ & $52(60)$ & & $50(58)$ & $37(42)$ & \\
\hline \multirow[t]{2}{*}{ Other loop invasion } & Absent & $115(50)$ & $115(50)$ & 1.000 & $149(65)$ & $81(35)$ & 0.192 \\
\hline & Present & $3(50)$ & $3(50)$ & & $2(33)$ & $4(67)$ & \\
\hline \multirow[t]{2}{*}{ Retroperitoneal seeding } & Absent & $110(50)$ & $111(50)$ & 0.790 & $145(66)$ & $76(34)$ & 0.046 \\
\hline & Present & $8(53)$ & $7(47)$ & & $6(40)$ & $9(60)$ & \\
\hline \multirow[t]{2}{*}{ Lymphovascular invasion } & Absent & $71(59)$ & $49(41)$ & 0.004 & $93(78)$ & $27(32)$ & $<0.001$ \\
\hline & Present & $47(40)$ & $69(60)$ & & $58(50)$ & $58(50)$ & \\
\hline \multirow[t]{2}{*}{ Perineural invasion } & Absent & $89(56)$ & $70(44)$ & 0.008 & $112(70)$ & $47(30)$ & 0.003 \\
\hline & Present & $29(38)$ & $48(62)$ & & $39(51)$ & $38(49)$ & \\
\hline \multirow[t]{2}{*}{ Resection margin status ${ }^{a}$} & No involvement & $107(50)$ & $106(50)$ & 1.000 & $136(64)$ & $77(36)$ & 0.727 \\
\hline & Cancer involvement & $4(44)$ & $5(56)$ & & $5(56)$ & $4(44)$ & \\
\hline Nodal metastasis ${ }^{\text {a }}$ & No & $73(66)$ & $38(34)$ & $<0.001$ & $84(76)$ & $27(24)$ & 0.001 \\
\hline
\end{tabular}


Table 2. Cont

\begin{tabular}{|c|c|c|c|c|c|c|c|}
\hline \multirow{2}{*}{\multicolumn{2}{|c|}{ Variable }} & \multicolumn{3}{|c|}{ рТВ, $N(\%)$} & \multicolumn{3}{|c|}{ іТВ, $N(\%)$} \\
\hline & & Low & High & $p$ & Low & High & $p$ \\
\hline \multirow{3}{*}{ T category ${ }^{b}$} & Yes & $40(37)$ & $68(63)$ & & $58(54)$ & $50(46)$ & \\
\hline & Low (T1-T2) & $26(93)$ & $2(7)$ & $<0.001$ & $25(89)$ & $3(11)$ & 0.002 \\
\hline & High (T3-T4) & $87(43)$ & $116(57)$ & & $121(60)$ & $82(40)$ & \\
\hline \multirow[t]{3}{*}{$\mathrm{N}$ category ${ }^{\mathrm{a}}$} & N0 & $73(66)$ & $38(34)$ & $<0.001$ & $84(76)$ & $27(24)$ & 0.003 \\
\hline & N1 & $18(33)$ & $37(67)$ & & $28(51)$ & $27(49)$ & \\
\hline & $\mathrm{N} 2$ & $22(41)$ & $31(59)$ & & $30(57)$ & $23(43)$ & \\
\hline \multirow[t]{2}{*}{ Stage grouping ${ }^{a, b}$} & Low (Stages I-II) & $68(64)$ & $38(36)$ & $<0.001$ & $79(75)$ & $27(25)$ & 0.002 \\
\hline & High (Stage III) & $40(37)$ & $68(63)$ & & $58(54)$ & $50(46)$ & \\
\hline \multirow[t]{2}{*}{ Chemotherapy ${ }^{a}$} & No & $82(55)$ & $67(45)$ & 0.105 & $100(67)$ & $49(33)$ & 0.139 \\
\hline & Yes & $36(44)$ & $46(56)$ & & $47(57)$ & $35(43)$ & \\
\hline \multirow[t]{2}{*}{ Radiation therapy ${ }^{a}$} & No & $109(54)$ & $94(46)$ & 0.047 & $135(67)$ & $68(33)$ & 0.025 \\
\hline & Yes & $9(33)$ & $18(67)$ & & $12(44)$ & $15(56)$ & \\
\hline \multirow[t]{2}{*}{$K R A S$ genotype ${ }^{\mathrm{a}}$} & Wild type & $59(46)$ & $70(54)$ & 0.282 & $80(62)$ & $49(38)$ & 0.254 \\
\hline & Mutated & $33(54)$ & $28(46)$ & & $43(71)$ & $18(29)$ & \\
\hline \multirow[t]{2}{*}{$B R A F$ genotype ${ }^{a}$} & Wild type & $85(48)$ & $91(52)$ & 1.000 & $115(65)$ & $61(35)$ & 1.000 \\
\hline & Mutated & $1(50)$ & $1(50)$ & & $1(50)$ & $1(50)$ & \\
\hline \multirow[t]{2}{*}{ MSI status ${ }^{a}$} & MSS & $98(53)$ & $87(47)$ & 0.060 & $121(65)$ & $64(35)$ & 0.334 \\
\hline & MSI-high & $19(38)$ & $31(62)$ & & $29(58)$ & $21(42)$ & \\
\hline
\end{tabular}

Significant $p$-values in bold; ${ }^{\text {a }}$ calculated using only patients with adequate data; ${ }^{\mathrm{b}}$ excluded patients with Tis and stage 0. 
In PDC, high levels of both pPDC and iPDC were also related to aggressiveness of SIACs: nodular or infiltrative growth pattern ( $p<0.001$ and $p=0.002$, respectively), high tumor grade $(p=0.002$ and $p<0.001$, respectively), nodal metastasis ( $p<0.001$ and $p=0.001$, respectively), higher T- $(p<0.001$ and $p=0.002$, respectively) and $\mathrm{N}$-categories ( $p<0.001$ and $p=0.003$, respectively) and stage grouping ( $p<0.001$ and $p=0.002$, respectively), and perineural ( $p=0.008$ and $p=0.003$, respectively) or lymphovascular ( $p=0.004$ and $p<0.001$, respectively) invasion. In addition, all PDC were observed in high levels in patients with nonintestinal type SIACs ( $p=0.029$ in pPDC; $p=0.018$ in iPDC) and radiotherapy $(p=0.047$ in $\mathrm{pPDC} ; p=0.025$ in iPDC). Meanwhile, SIACs with high pPDC more frequently had pancreatic invasion $(p=0.022)$, while those with high iPDC had retroperitoneal seeding $(p=0.046)$. In addition, pPDC was higher in older patients $(p=0.009)$. KRAS and BRAF mutations and MSI status were not associated with TB and PDC in SIAC.

\subsection{Associations between $p T B, i T B, p P D C$, and $i P D C$}

To focus our investigations on pTB, we assessed its correlation with iTB, pPDC, and iPDC (Table 3). In a multivariate logistic regression analysis, high pTB remained significantly more frequent in SIACs with high levels of iTB (odds ratio, 69.203; 95\% confidence interval, 15.586-307.264) and pPDC (odds ratio, 17.294; $95 \%$ confidence interval, 5.932-50.420) $(p<0.001$, both). There was no relationship between $\mathrm{pTB}$ and $\operatorname{PDC}(p=0.291)$.

Table 3. Association between pTB Status and iTB, pPDC, and iPDC in SIAC

\begin{tabular}{ccccccc}
\hline \multirow{2}{*}{ Variable } & \multicolumn{2}{c}{ Frequency Analysis, $N$ (\%) } & \multicolumn{2}{c}{ Multivariate Logistic Regression Analysis } \\
\cline { 3 - 7 } & & Low pTB & High pTB & $p$ & OR (95\% CI) & $p$ \\
\hline \multirow{2}{*}{ iTB } & Low & $60(56)$ & $47(44)$ & $<\mathbf{0 . 0 0 1}$ & $69.203(15.586-307.264)$ & $<\mathbf{0 . 0 0 1}$ \\
& High & $2(2)$ & $127(98)$ & & & $<$ \\
\multirow{2}{*}{ pPDC } & Low & $57(48)$ & $61(52)$ & $<\mathbf{0 . 0 0 1}$ & $17.294(5.932-50.420)$ & $<0.001$ \\
& High & $5(4)$ & $113(96)$ & & & 0.291 \\
\multirow{2}{*}{ iPDC } & Low & $59(39)$ & $92(61)$ & $<\mathbf{0 . 0 0 1}$ & $2.359(0.479-11.609)$ & \\
& High & $3(3)$ & $82(97)$ & & & \\
\hline
\end{tabular}

TB at the invasive front (pTB); TB in the tumor center (iTB); PDC at the invasive front (pPDC); PDC in the tumor center (iPDC); odds ratio (OR); confidence interval (CI); significant $p$-values in bold.

\subsection{Survival Analysis}

The results of the survival analyses of TB and PDC are described in Figure 2. Patients with high pTB (median: 23.3 months) demonstrated significantly worse overall survival than those with low pTB (133.7 months; $p<0.001$, Figure 2A). Patients with high iTB (23.3 months) also had a shorter survival time than those with low iTB (50.1 months; $p=0.001$, Figure 2B). In pPDC, a high level (24.9 months) was significantly associated with worse overall survival of patients than a low level (47.4 months; $p=0.023$, Figure 2C). In addition, SIAC patients with high iPDC (23.0 months) showed worse survival than those with low iPDC (44.4 months; $p=0.043$, Figure 2D). The following clinicopathologic factors were also associated with worse SIAC patient survival by univariate analysis (Table 4): nonintestinal phenotype $(p<0.001)$, distal location $(p=0.004)$, nodal metastasis $(p<0.001)$, higher T- and N-categories $(p=0.002$ and $p<0.001$, respectively) and stage groupings $(p<0.001)$, presence of retroperitoneal seeding $(p<0.001)$ and perineural and lymphovascular invasions $(p=0.004$ and $p<0.001$, respectively), radiotherapy $(p=0.008)$, and MSS $(p=0.029)$. 

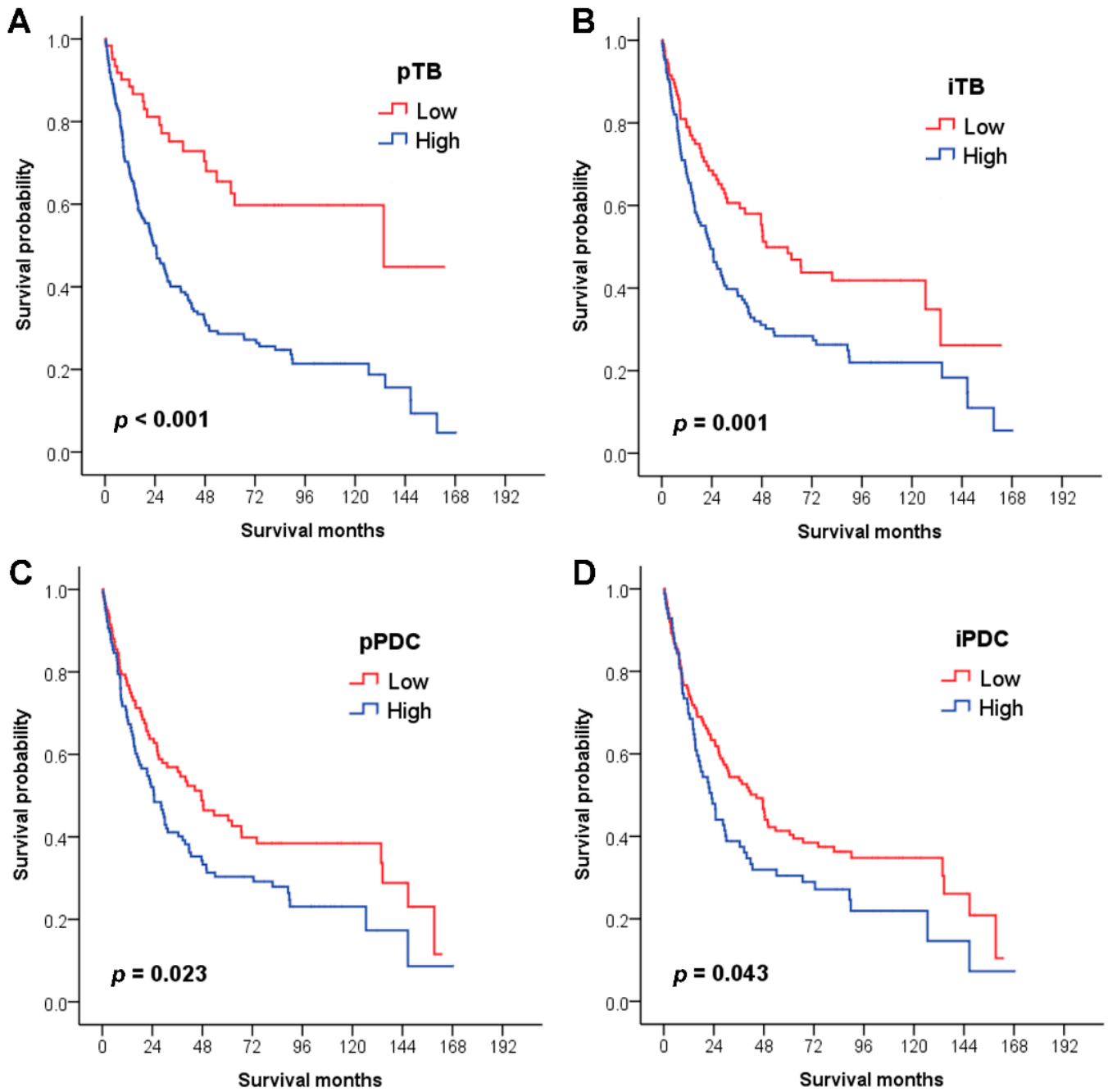

Figure 2. Survival analysis based on the status of TB and PDC. (A) SIAC patients with high pTB (median: 23.3 months) demonstrate significantly worse overall survival than those with low pTB (133.7 months; $p$ 0.001). (B) SIAC patients with high iTB (23.3 months) also have a shorter survival time than those with low iTB (50.1 months; $p=0.001)$. (C) SIAC patients with a high pPDC level (24.9 months) demonstrate significantly worse overall survival than those with a low level (47.4 months; $p=0.023$ ). In addition, (D) SIAC patients with high iPDC (23.0 months) have worse survival than those with low iPDC (44.4 months; $p=0.043)$.

Multivariate analysis revealed that high pTB $(p=0.024)$, nonintestinal type $(p=0.001)$, high N-category $(p=0.001)$, retroperitoneal seeding $(p=0.039)$, and MSS $(p=0.003)$ were worse independent prognostic predictors (Table 4).

\subsection{Survival Analysis of $p T B$ based on Stage Groups}

The independent prognostic significance of PTB in SIACs, which was considered significant by multivariate analysis, was further evaluated according to stage (Figure 3 ). In the lower stage (stages I-II) group $(n=106)$, the overall survival times for SIAC patients with high pTB $(n=63$; median: 42.5 months) were significantly shorter than those with low pTB $(n=43 ; 133.7$ months; $p=0.007$, Figure $3 \mathrm{~A})$. However, in the higher stage group $(n=108)$, there was no statistical significance in survival time distribution between the patients with high pTB $(n=97 ; 21.0$ months $)$ and those with low $\operatorname{pTB}(n=11 ; 37.4$ months; $p=0.125$, Figure 3B). 
Table 4. Univariate and Multivariate Analyses of SIAC Patients.

\begin{tabular}{|c|c|c|c|c|c|c|}
\hline \multirow{2}{*}{ Variable } & & \multicolumn{3}{|c|}{ Univariate } & \multicolumn{2}{|c|}{ Multivariate } \\
\hline & & Median Survival (mo) & HR $(95 \%$ CI) & $p$ & HR $(95 \%$ CI) & $p$ \\
\hline \multirow[t]{2}{*}{ pTB } & Low & 133.7 & & & & \\
\hline & High & 23.3 & 2.947 (1.858-4.675) & $<0.001$ & $1.890(1.086-3.289)$ & 0.024 \\
\hline \multirow[t]{2}{*}{ iTB } & Low & 50.1 & & & & \\
\hline & High & 23.3 & $1.711(1.226-2.386)$ & 0.001 & & \\
\hline \multirow[t]{2}{*}{ pPDC } & Low & 47.4 & & & & \\
\hline & High & 24.9 & $1.450(1.051-2.001)$ & 0.023 & & \\
\hline \multirow[t]{2}{*}{ iPDC } & Low & 44.4 & & & & \\
\hline & High & 23.0 & $1.398(1.010-1.935)$ & 0.043 & & \\
\hline \multirow[t]{2}{*}{ Location } & Proximal & 41.7 & & & & \\
\hline & Distal & 22.5 & $1.598(1.157-2.206)$ & 0.004 & $1.455(0.992-2.134)$ & 0.055 \\
\hline \multirow[t]{2}{*}{ Immunophenotype ${ }^{\text {a }}$} & Intestinal & 134.4 & & & & \\
\hline & Nonintestinal & 22.0 & 2.533 (1.701-3.773) & $<0.001$ & 1.999 (1.309-3.053) & 0.001 \\
\hline \multirow[t]{2}{*}{ Retroperitoneal seeding } & Absent & 37.4 & & & & \\
\hline & Present & 14.0 & $2.997(1.714-5.242)$ & $<0.001$ & 2.079 (1.037-4.166) & 0.039 \\
\hline \multirow[t]{2}{*}{ Lymphovascular invasion } & Absent & 66.6 & & & & \\
\hline & Present & 17.8 & $2.357(1.695-3.277)$ & $<0.001$ & $1.476(0.971-2.242)$ & 0.068 \\
\hline \multirow[t]{2}{*}{ Perineural invasion } & Absent & 47.6 & & & & \\
\hline & Present & 17.8 & $1.635(1.172-2.281)$ & 0.004 & $1.051(0.707-1.562)$ & 0.805 \\
\hline \multirow[t]{2}{*}{ Nodal metastasis ${ }^{a}$} & Absent & 133.7 & & & & \\
\hline & Present & 21.6 & $2.401(1.690-3.411)$ & $<0.001$ & & \\
\hline \multirow[t]{2}{*}{ T category ${ }^{b}$} & Low (T1-T2) & $-{ }^{c}$ & & & & \\
\hline & High (T3-T4) & 26.3 & $3.167(1.553-6.458)$ & 0.002 & $1.128(0.484-2.628)$ & 0.780 \\
\hline \multirow[t]{4}{*}{$\mathrm{N}$ category ${ }^{\mathrm{a}}$} & & & & $<0.001$ & & 0.001 \\
\hline & No & 133.7 & & & 1 & - \\
\hline & N1 & 28.4 & $1.987(1.312-3.008)$ & 0.001 & $1.445(0.903-2.313)$ & 0.125 \\
\hline & $\mathrm{N} 2$ & 17.3 & $2.981(1.981-4.486)$ & $<0.001$ & $2.501(1.549-4.036)$ & $<0.001$ \\
\hline \multirow[t]{2}{*}{ Stage grouping $\mathrm{a}, \mathrm{b}$} & Low (Stages I-II) & 133.7 & & & & \\
\hline & High (Stage III) & 21.6 & $2.342(1.645-3.335)$ & $<0.001$ & & \\
\hline \multirow[t]{2}{*}{ Radiation therapy $^{\mathrm{a}}$} & $\mathrm{No}$ & 38.5 & & & & \\
\hline & Yes & 23.3 & $1.824(1.172-2.839)$ & 0.008 & $0.935(0.572-1.528)$ & 0.789 \\
\hline \multirow[t]{2}{*}{ MSI status ${ }^{a}$} & MSS & 28.2 & & & & \\
\hline & MSI-high & 72.6 & $0.629(0.414-0.954)$ & 0.029 & $0.470(0.287-0.769)$ & 0.003 \\
\hline
\end{tabular}

Hazard ratio (HR); confidence interval (CI); significant $p$-values in bold; ${ }^{a}$ calculated using only patients with adequate data; ${ }^{\mathrm{b}}$ excluded patients with Tis and stage $0 ;{ }^{\mathrm{c}}$ cannot be calculated because $>50 \%$ of patients were alive. 
A

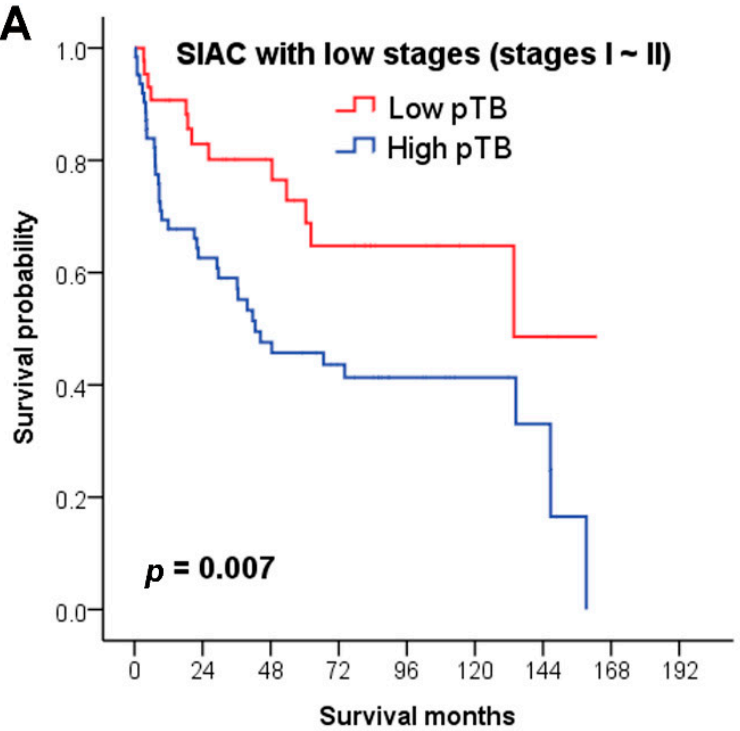

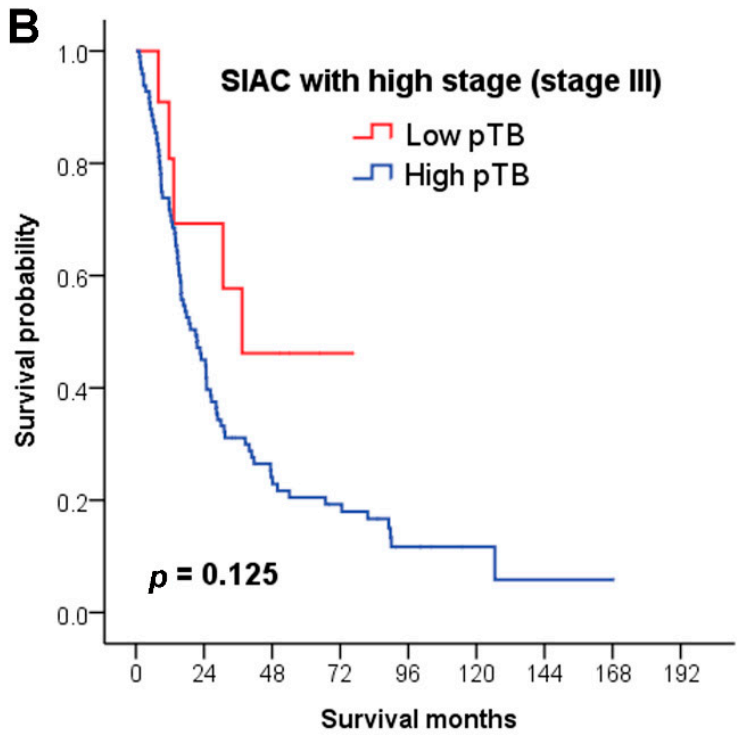

Figure 3. Survival analysis of pTB based on stage group. (A) In the lower stage (stages I-II) group $(n=106)$, the overall survival times for SIAC patients with high pTB ( $n=63$; median: 42.5 months $)$ is significantly shorter than those with low pTB ( $n=43 ; 133.7$ months; $p=0.007)$. However, $(\mathbf{B})$ in the higher stage group $(n=108)$, there is no statistical significance in survival time distribution between the patients with high $\operatorname{pTB}(n=97 ; 21.0$ months $)$ and those with low pTB $(n=11 ; 37.4$ months; $p=0.125)$.

\section{Discussion}

To the best of our knowledge, our study is the first to report the clinicopathologic significance of TB and PDC and their prognostic values in SIACs. In CRC, pTB and iTB have been well-known indicators linked to higher TNM stage and tumor grade, lymphovascular invasion, or nodal and distant metastases $[13,22,24]$. Furthermore, the similarities in adverse prognostic roles of pTB and iTB were described in a study by Lugli and colleagues [23]. In the present study, we observed that high levels of pTB and iTB were associated with aggressiveness of SIACs such as high tumor grade, higher T- and N-categories and stage grouping, nodular or infiltrative growth pattern, perineural or lymphovascular invasion, and pancreatic invasion. Moreover, SIAC patients with high pTB and/or iTB had significantly shorter overall survival. These findings are concordant with those of previous studies in CRC. However, the clinical impact of iTB was somewhat different from pTB. iTB seemed to qualify for investigation in the prospective setting [24]; for example, high iTB rate in the preoperative biopsy of CRC patients could be predictive of nodal and distant metastases [24]. Therefore, in endoscopically resected pT1 CRC, iTB could help to select the patients who required surgical resection due to a high risk of nodal and distant metastasis $[13,34]$. In addition, iTB in preoperative biopsies could identify CRC patients who may be eligible for neoadjuvant chemoradiotherapy and potentially predict tumor regression [13,35]. In subgroups of nodal negative CRC, iTB could also select the patients who might show aggressive behavior and benefit from adjuvant therapy [22]. Our findings suggest that iPDC seemed to have a similar biological significance to iTB, since iPDC in the preoperative biopsy could predict the metastatic risk of patients [25] as well as tumor response and clinical outcome in CRC treated with neoadjuvant chemoradiotherapy [36]. Unfortunately, we could not include the preoperative biopsy specimens and the detailed information about chemotherapy in this study owing to the limitation of a multi-institutional study. Therefore, to analyze the utility of iTB and/or iPDC in biopsy specimens for the therapeutic response and prognosis of patients with SIAC, further investigations that include patients treated with modern treatment strategies after biopsy are necessary.

We graded PDC according to a proposal by Ueno et al., which was identical to the TB grading system [28]. In the previous CRC study by Ueno and colleagues, pPDC was a prognostic indicator with more accuracy than PTB [28]. However, the prognostic effect of pTB might be obscured due 
to selection bias because the $\mathrm{T} 1$ category was excluded [28]. In the present study, we assessed the prognostic significances of $\mathrm{pTB}, \mathrm{iTB}, \mathrm{pPDC}$, and iPDC independently by multivariate analysis as all of them were related to the survival of patients with SIAC. Among them, we found that pTB provided a significant predictive value, whereas the others did not (iTB, $p=0.675$; $\mathrm{pPDC}, p=0.932$; $\mathrm{iPDC}, p=0.291$.). In addition, we identified a prognostic value of $\mathrm{pTB}$ for survival in the lower stage (stages I-II) group of SIAC. Concordant with our results, Prall et al. found an adverse prognostic effect of pTB, specifically in a series of stage I and II CRC patients $(n=186)$ [37]. Therefore, pTB status could be used as an additional prognostic predictor in surgically resected SIAC patients with lower disease stage. Moreover, we observed that SIAC patients with high pTB had worse overall survival than those with low pTB in the stage III group, but it was not statistically significant. Stage IV was not included in this study; thus, further studies with larger numbers of cases with stage IV disease are required to support the use of $\mathrm{PTB}$ as an adverse prognosticator even in high stages.

Both TB and PDC are understood as EMT phenomena, activated by the Wnt $/ \beta$-catenin signaling pathway [38]. TB acquires a mesenchymal phenotype with nuclear translocation of $\beta$-catenin and concomitant loss of membranous E-cadherin expression resulting in the activation of the Wnt pathway $[13,18]$. In PDC, the L1 cell adhesion molecule (L1CAM), which generates epithelial cell migration as one of the target factors of the Wnt signaling pathway, was upregulated [38,39]. In addition, oncogenic RAS mutation was associated with high PDC in CRCs [31,40]. In the present study, we could not find any significant association between KRAS mutation with TB and PDC in SIACs. To evaluate the association with TB and/or PDC and immune-phenotypes of SIACs, we compared our results of TB and PDC with that of our previous study on CDX2 and MUC1 immunolabeling [33]. As is known, CDX2 expression is related to the intestinal phenotype, while MUC1 is mainly expressed in the pancreatic duct and superficial foveolar epithelium [33]. As a result, we could identify that the loss of intestinal differentiation exhibiting CDX2- and/or MUC1+ was related to high levels of TB and PDC in SIACs. Moreover, the nonintestinal immuno-phenotype was also revealed as an independent worse prognostic parameter. Therefore, it might be useful to examine CDX2 and MUC1 immunolabeling patterns as intestinal versus nonintestinal in biopsy specimens to predict the prognosis of a patient with SIAC since pTB cannot be evaluated in biopsy specimens without evaluating the invasion front.

Cancer cells can invade surrounding structures as cell clusters as well as through single-cell invasion [41]. In a CRC study using Ezrin immunolabeling, PDC could serve as a platform for converting the migration manner from collective-cell to single-cell invasion [42]. Three-dimensional reconstruction of $4 \mu \mathrm{m}$ serially sectioned immunohistochemical staining slides of solid cancer, including CRCs, revealed that TB was connected with the main tumor mass [43]. Similarly, TB in isolation in one plane of a section could be traced to the neighboring neoplasm at the invasion front by electron microscopy and immunohistochemical staining [44]. These findings may represent sequential steps in tumor mass outgrowth via PDC to TB. TB may evolve from PDC by acquiring proliferative and aggregative activities [45].

The present study has some limitations. We selected only a single slide containing the deepest invasion front for evaluating TB and PDC, although all $\mathrm{H}$ and $\mathrm{E}$ slides were initially assessed to select a representative slide. However, a previous study demonstrated an excellent agreement between the multi-slide and single-slide methods of analysis for TB and PDC in CRCs using a Japanese $(n=283)$ and a Scottish $(n=163)$ cohort. Herein, the analysis from a single representative $\mathrm{H}$ and $\mathrm{E}$ slide evaluation containing the deepest invasion front was an efficient way of evaluating TB and PDC [46].

\section{Conclusions}

High levels of TB and PDC, both in peritumoral and intratumoral regions, were associated with aggressive behavior and poor survival outcomes of patients with SIAC. Among TB and PDC, pTB can be used as a powerful independent adverse prognostic indicator in patients with SIACs, especially when patients are in early disease stages (stage I or II). 
Author Contributions: Conceptualization, S.-Y.J. and S.-M.H.; methodology, S.-Y.J.; software, J.-Y.C.; investigation, S.-Y.J. and N.Y.; data curation and formal analysis, J.-Y.C. and N.Y.; resources, J.-Y.C., E.S.J., and Y.-H.O.; writing—original draft preparation, S.-Y.J.; writing—review and editing, S.-M.H.; visualization, S.-Y.J.; supervision, S.-M.H.; project administration and funding acquisition, S.-Y.J. All authors have read and agreed to the published version of the manuscript.

Funding: This research was supported by the Basic Science Research Program through the National Research Foundation of Korea (NRF) funded by the Ministry of Education (NRF-2017R1D1A1B03031817, awarded to S.-Y.J.).

Acknowledgments: This work was presented in part at the 2020 annual meeting of the United States and Canadian Academy of Pathology in Los Angeles, California, USA, 28th February-5th March 2020. We would like to thank the members of the Korean Small Intestinal Cancer Study Group for providing samples and data for this study: Hee-Kyung Chang, Kosin University College of Medicine, Pusan; Kee-Taek Jang, Samsung Medical Center, Sungkyunkwan University School of Medicine, Seoul; Young Kyung Bae, Yeungnam University College of Medicine, Daegu; Ghil Suk Yoon, Kyungpook National University School of Medicine, Dague; Joon Mee Kim, Inha University College of Medicine, Incheon; Gwang Il Kim, CHA Bundang Medical Center, CHA University, Seongnam; Hee Kyung Kim, Soonchunhyang University Bucheon Hospital, Bucheon; Kyu Yun Jang, Chonbuk National University Medical School, Jeonju; Dae Woon Eom, University of Ulsan College of Medicine, Gangneung Asan Hospital, Gangneung; Jae Bok Park, Catholic University of Daegu, Daegu; Soo Jin Jung, Inje University College of Medicine, Busan; Gyeong Hoon Kang, Seoul National University College of Medicine, Seoul; Ji Shin Lee, Chonnam National University Medical School, Gwangju; Kye Won Kwon, Bundang Jesaeng General Hospital, Seongnam; Soon Won Hong, Yonsei University College of Medicine, Seoul; Han-Ik Bae, Kyungpook National University School of Medicine, Dague; Hyun-Jung Kim, Inje University Sanggye Paik Hospital, Seoul; Jinwon Seo, Hallym University Sacred Heart Hospital, Anyang; and Eun Kyoung Kwak, Fatima Hospital, Daegu, Korea.

Conflicts of Interest: The authors declare no conflict of interest.

\section{References}

1. Klimstra, D.; Nagteggal, I.; Rugge, M.; Salto-Tellez, M. Tumours of the small intestine and ampulla. In WHO Classification of Tumours: Digestive System Tumours, 5th ed.; Carneiro, F., Ochiai, A., Chan, J., Oliva, E., Cheung, N.-Y., Rous, B., Cree, I., Singh, R., Fitzgibbons, P., Soares, F., et al., Eds.; IARC: Lyon, France, 2019; pp. 111-134.

2. Bilimoria, K.Y.; Bentrem, D.J.; Wayne, J.D.; Ko, C.Y.; Bennett, C.L.; Talamonti, M.S. Small bowel cancer in the United States: Changes in epidemiology, treatment, and survival over the last 20 years. Ann. Surg. 2009, 249, 63-71. [CrossRef] [PubMed]

3. Siegel, R.L.; Miller, K.D.; Jemal, A. Cancer statistics, 2020. CA Cancer J. Clin. 2020, 70, 7-30. [CrossRef] [PubMed]

4. Annual Report of Cancer Statistics in Korea in 2016. Available online: http://ncc.re.kr/cancerStatsList.ncc? searchKey=total\&searchValue=\&pageNum $=1$ (accessed on 15 April 2020).

5. Young, J.I.; Mongoue-Tchokote, S.; Wieghard, N.; Mori, M.; Vaccaro, G.M.; Sheppard, B.C.; Tsikitis, V.L. Treatment and survival of small-bowel adenocarcinoma in the United States: A comparison with colon cancer. Dis. Colon Rectum 2016, 59, 306-315. [CrossRef] [PubMed]

6. Pedersen, K.S.; Raghav, K.; Overman, M.J. Small bowel adenocarcinoma: Etiology, presentation, and molecular alterations. J. Natl. Compr. Cancer Netw. 2019, 17, 1135-1141. [CrossRef]

7. Benson, A.B.; Venook, A.P.; Al-Hawary, M.M.; Arain, M.A.; Chen, Y.J.; Ciombor, K.K.; Cohen, S.A.; Cooper, H.S.; Deming, D.A.; Garrido-Laguna, I.; et al. Small bowel adenocarcinoma, version 1.2020, NCCN clinical practice guidelines in oncology. J. Natl. Compr. Cancer Netw. 2019, 17, 1109-1133. [CrossRef]

8. Schrock, A.B.; Devoe, C.E.; McWilliams, R.; Sun, J.; Aparicio, T.; Stephens, P.J.; Ross, J.S.; Wilson, R.; Miller, V.A.; Ali, S.M.; et al. Genomic profiling of small-bowel adenocarcinoma. JAMA Oncol. 2017, 3, 1546-1553. [CrossRef]

9. Jun, S.Y.; Park, E.S.; Lee, J.J.; Chang, H.K.; Jung, E.S.; Oh, Y.H.; Hong, S.M. Prognostic significance of stromal and intraepithelial tumor-infiltrating lymphocytes in small intestinal adenocarcinoma. Am. J. Clin. Pathol. 2020, 153, 105-118. [CrossRef]

10. Chang, H.K.; Yu, E.; Kim, J.; Bae, Y.K.; Jang, K.T.; Jung, E.S.; Yoon, G.S.; Kim, J.M.; Oh, Y.H.; Bae, H.I.; et al. Adenocarcinoma of the small intestine: A multi-institutional study of 197 surgically resected cases. Hum. Pathol. 2010, 41, 1087-1096. [CrossRef] 
11. Jun, S.Y.; Kim, M.; Gu, M.J.; Bae, Y.K.; Chang, H.K.; Jung, E.S.; Jang, K.T.; Kim, J.; Yu, E.; Eom, D.W.; et al. Clinicopathologic and prognostic associations of KRAS and BRAF mutations in small intestinal adenocarcinoma. Mod. Pathol. 2016, 29, 402-415. [CrossRef]

12. Kim, A.; Bae, Y.K.; Gu, M.J.; Kim, J.Y.; Jang, K.Y.; Bae, H.I.; Lee, H.J.; Hong, S.M. Epithelial-mesenchymal transition phenotype is associated with patient survival in small intestinal adenocarcinoma. Pathology 2013, 45, 567-573. [CrossRef]

13. Lugli, A.; Kirsch, R.; Ajioka, Y.; Bosman, F.; Cathomas, G.; Dawson, H.; El Zimaity, H.; Flejou, J.F.; Hansen, T.P.; Hartmann, A.; et al. Recommendations for reporting tumor budding in colorectal cancer based on the International Tumor Budding Consensus Conference (ITBCC) 2016. Mod. Pathol. 2017, 30, 1299-1311. [CrossRef] [PubMed]

14. Zlobec, I.; Lugli, A. Epithelial mesenchymal transition and tumor budding in aggressive colorectal cancer: Tumor budding as oncotarget. Oncotarget 2010, 1, 651-661. [CrossRef] [PubMed]

15. Nagtegaal, I.; Arends, M.; Odze, R.; Lam, A. Tumours of the colon and rectum. In WHO Classification of Tumours: Digestive System Tumours, 5th ed.; Carneiro, F., Ochiai, A., Chan, J., Oliva, E., Cheung, N.-Y., Rous, B., Cree, I., Singh, R., Fitzgibbons, P., Soares, F., et al., Eds.; IARC: Lyon, France, 2019; pp. 157-192.

16. Jesinghaus, M.; Bruhl, F.; Steiger, K.; Klare, P.; Reiser, M.; Scheiter, A.; Konukiewitz, B.; Kuhn, P.; Munch, S.; Quante, M.; et al. Cellular dissociation grading based on the parameters tumor budding and cell nest size in pretherapeutic biopsy specimens allows for prognostic patient stratification in esophageal squamous cell carcinoma independent from clinical staging. Am. J. Surg. Pathol. 2019, 43, 618-627. [CrossRef] [PubMed]

17. Thies, S.; Guldener, L.; Slotta-Huspenina, J.; Zlobec, I.; Koelzer, V.H.; Lugli, A.; Kroll, D.; Seiler, C.A.; Feith, M.; Langer, R. Impact of peritumoral and intratumoral budding in esophageal adenocarcinomas. Hum. Pathol. 2016, 52, 1-8. [CrossRef]

18. Koelzer, V.H.; Langer, R.; Zlobec, I.; Lugli, A. Tumor budding in upper gastrointestinal carcinomas. Front. Oncol. 2014, 4, 216. [CrossRef]

19. Landau, M.S.; Hastings, S.M.; Foxwell, T.J.; Luketich, J.D.; Nason, K.S.; Davison, J.M. Tumor budding is associated with an increased risk of lymph node metastasis and poor prognosis in superficial esophageal adenocarcinoma. Mod. Pathol. 2014, 27, 1578-1589. [CrossRef]

20. Kemi, N.; Eskuri, M.; Ikalainen, J.; Karttunen, T.J.; Kauppila, J.H. Tumor budding and prognosis in gastric adenocarcinoma. Am. J. Surg. Pathol. 2019, 43, 229-234. [CrossRef]

21. Du, M.; Chen, L.; Cheng, Y.; Wang, Y.; Fan, X.; Zhang, Y.; Zhou, X.; Guo, L.; Xu, G.; Zou, X.; et al. Tumor budding and other risk factors of lymph node metastasis in submucosal early gastric carcinoma: A multicenter clinicopathologic study in 621 radical gastrectomies of chinese patients. Am. J. Surg. Pathol. 2019, 43, 1074-1082. [CrossRef]

22. Marx, A.H.; Mickler, C.; Sauter, G.; Simon, R.; Terracciano, L.M.; Izbicki, J.R.; Clauditz, T.S. High-grade intratumoral tumor budding is a predictor for lymphovascular invasion and adverse outcome in stage II colorectal cancer. Int. J. Colorectal Dis. 2020, 35, 259-268. [CrossRef]

23. Lugli, A.; Vlajnic, T.; Giger, O.; Karamitopoulou, E.; Patsouris, E.S.; Peros, G.; Terracciano, L.M.; Zlobec, I. Intratumoral budding as a potential parameter of tumor progression in mismatch repair-proficient and mismatch repair-deficient colorectal cancer patients. Hum. Pathol. 2011, 42, 1833-1840. [CrossRef]

24. Zlobec, I.; Hadrich, M.; Dawson, H.; Koelzer, V.H.; Borner, M.; Mallaev, M.; Schnuriger, B.; Inderbitzin, D.; Lugli, A. Intratumoural budding (ITB) in preoperative biopsies predicts the presence of lymph node and distant metastases in colon and rectal cancer patients. Br. J. Cancer 2014, 110, 1008-1013. [CrossRef] [PubMed]

25. Barresi, V.; Bonetti, L.R.; Ieni, A.; Branca, G.; Baron, L.; Tuccari, G. Histologic grading based on counting poorly differentiated clusters in preoperative biopsy predicts nodal involvement and pTNM stage in colorectal cancer patients. Hum. Pathol. 2014, 45, 268-275. [PubMed]

26. Barresi, V.; Reggiani Bonetti, L.; Ieni, A.; Caruso, R.A.; Tuccari, G. Poorly differentiated clusters: Clinical impact in colorectal cancer. Clin. Colorectal Cancer 2017, 16, 9-15. [CrossRef] [PubMed]

27. Ueno, H.; Mochizuki, H.; Hashiguchi, Y.; Ishiguro, M.; Kajiwara, Y.; Sato, T.; Shimazaki, H.; Hase, K.; Talbot, I.C. Histological grading of colorectal cancer: A simple and objective method. Ann. Surg. 2008, 247, 811-818. [CrossRef]

28. Ueno, H.; Kajiwara, Y.; Shimazaki, H.; Shinto, E.; Hashiguchi, Y.; Nakanishi, K.; Maekawa, K.; Katsurada, Y.; Nakamura, T.; Mochizuki, H.; et al. New criteria for histologic grading of colorectal cancer. Am. J. Surg. Pathol. 2012, 36, 193-201. [CrossRef] 
29. Barresi, V.; Reggiani Bonetti, L.; Branca, G.; Di Gregorio, C.; Ponz de Leon, M.; Tuccari, G. Colorectal carcinoma grading by quantifying poorly differentiated cell clusters is more reproducible and provides more robust prognostic information than conventional grading. Virchows Arch. 2012, 461, 621-628. [CrossRef]

30. Bertoni, L.; Barresi, V.; Bonetti, L.R.; Caramaschi, S.; Mangogna, A.; Lionti, S.; Azzoni, P.; Carnevale, G.; Pisciotta, A.; Salviato, T. Poorly differentiated clusters (PDC) in colorectal cancer: Does their localization in tumor matter? Ann. Diagn. Pathol. 2019, 41, 106-111. [CrossRef]

31. Reggiani Bonetti, L.; Barresi, V.; Bettelli, S.; Caprera, C.; Manfredini, S.; Maiorana, A. Analysis of KRAS, NRAS, PIK3CA, and BRAF mutational profile in poorly differentiated clusters of KRAS-mutated colon cancer. Hum. Pathol. 2017, 62, 91-98. [CrossRef]

32. Edge, S.B.; Greene, F.L.; Schilsky, R.L.; Gaspar, L.E.; Washington, M.K.; Sullivan, D.C.; Brookland, R.K.; Brierley, J.D.; Balch, C.M.; Campton, C.C.; et al. AJCC Cancer Staging Manual, 8th ed.; Springer Nature: Basel, Switzerland, 2017; pp. 221-234.

33. Jun, S.Y.; Eom, D.W.; Park, H.; Bae, Y.K.; Jang, K.T.; Yu, E.; Hong, S.M. Prognostic significance of CDX2 and mucin expression in small intestinal adenocarcinoma. Mod. Pathol. 2014, 27, 1364-1374. [CrossRef]

34. Koelzer, V.H.; Zlobec, I.; Lugli, A. Tumor budding in colorectal cancer-ready for diagnostic practice? Hum. Pathol. 2016, 47, 4-19. [CrossRef]

35. Rogers, A.C.; Gibbons, D.; Hanly, A.M.; Hyland, J.M.; O'Connell, P.R.; Winter, D.C.; Sheahan, K. Prognostic significance of tumor budding in rectal cancer biopsies before neoadjuvant therapy. Mod. Pathol. 2014, 27, 156-162. [CrossRef] [PubMed]

36. Reggiani Bonetti, L.; Lionti, S.; Domati, F.; Pagliani, G.; Mattioli, E.; Barresi, V. Histological grading based on poorly differentiated clusters is predictive of tumour response and clinical outcome in rectal carcinoma treated with neoadjuvant chemoradiotherapy. Histopathology 2017, 71, 393-405. [CrossRef] [PubMed]

37. Prall, F.; Nizze, H.; Barten, M. Tumour budding as prognostic factor in stage I/II colorectal carcinoma. Histopathology 2005, 47, 17-24. [CrossRef]

38. Yonemura, K.; Kajiwara, Y.; Ao, T.; Mochizuki, S.; Shinto, E.; Okamoto, K.; Hase, K.; Ueno, H. Prognostic value of poorly differentiated clusters in liver metastatic lesions of colorectal carcinoma. Am. J. Surg. Pathol. 2019, 43, 1341-1348. [CrossRef]

39. Kajiwara, Y.; Ueno, H.; Hashiguchi, Y.; Shinto, E.; Shimazaki, H.; Mochizuki, H.; Hase, K. Expression of L1 cell adhesion molecule and morphologic features at invasive front of colorectal cancer. Am. J. Clin. Pathol. 2011, 136, 138-144. [CrossRef]

40. Barresi, V.; Bonetti, L.R.; Bettelli, S. KRAS, NRAS, BRAF mutations and high counts of poorly differentiated clusters of neoplastic cells in colorectal cancer: Observational analysis of 175 cases. Pathology 2015, 47, 551-556. [CrossRef] [PubMed]

41. Friedl, P.; Locker, J.; Sahai, E.; Segall, J.E. Classifying collective cancer cell invasion. Nat. Cell Biol. 2012, 14, 777-783. [CrossRef] [PubMed]

42. Aikawa, A.; Fujita, H.; Kosaka, T.; Minato, H.; Kiyokawa, E. Clinicopathological significance of heterogeneic ezrin expression in poorly differentiated clusters of colorectal cancers. Cancer Sci. 2019, 110, 2667-2675.

43. Enderle-Ammour, K.; Wellner, U.; Kocsmar, E.; Kiss, A.; Lotz, G.; Csanadi, A.; Bader, M.; Schilling, O.; Werner, M.; Bronsert, P. Three-dimensional reconstruction of solid tumors: Morphological evidence for tumor heterogeneity. Pathologe 2018, 39, 231-235. [CrossRef]

44. Prall, F.; Ostwald, C.; Linnebacher, M. Tubular invasion and the morphogenesis of tumor budding in colorectal carcinoma. Hum. Pathol. 2009, 40, 1510-1512. [CrossRef]

45. Fonseca, G.M.; de Mello, E.S.; Faraj, S.F.; Kruger, J.A.P.; Coelho, F.F.; Jeismann, V.B.; Lupinacci, R.M.; Cecconello, I.; Alves, V.A.F.; Pawlik, T.M.; et al. Prognostic significance of poorly differentiated clusters and tumor budding in colorectal liver metastases. J. Surg. Oncol. 2018, 117, 1364-1375. [CrossRef] [PubMed]

46. Nearchou, I.P.; Kajiwara, Y.; Mochizuki, S.; Harrison, D.J.; Caie, P.D.; Ueno, H. Novel internationally verified method reports desmoplastic reaction as the most significant prognostic feature for disease-specific survival in stage II colorectal cancer. Am. J. Surg. Pathol. 2019, 43, 1239-1248. [CrossRef] [PubMed]

(C) 2020 by the authors. Licensee MDPI, Basel, Switzerland. This article is an open access article distributed under the terms and conditions of the Creative Commons Attribution (CC BY) license (http://creativecommons.org/licenses/by/4.0/). 